\title{
No time-stretching illusion when a tone is followed by a noise
}

\author{
Tsuyoshi Kuroda $\cdot$ Simon Grondin
}

Published online: 12 September 2013

(C) Psychonomic Society, Inc. 2013

\begin{abstract}
A sine tone is perceived as longer when it is preceded by a more intense noise than when presented in isolation. This is the time-stretching illusion. We conducted an experiment where the method of constant stimuli was used to examine whether a tone would also be stretched when it was followed by a noise. The duration of a tone was overestimated when it was preceded by a noise, but not when followed by a noise or when located between two consecutive noises. Moreover, the increasing of the noise intensity (from -6 to $+6 \mathrm{~dB}$ ) relative to the tone intensity resulted in larger overestimations, but only in the condition where a tone was preceded by a noise. In brief, the duration of a tone is stretched when this tone is preceded by a noise and if this tone is not followed by a noise.
\end{abstract}

Keywords Auditory illusion · Filled duration ·

Discrimination $\cdot$ Sound edge $\cdot$ Intensity

A sine tone is perceived as longer when it is preceded by a more intense noise than when presented in isolation (Fig. 1a). This phenomenon was originally reported by Sasaki, Nakajima, and ten Hoopen (1993; see also ten Hoopen, Miyauchi, \& Nakajima, 2008). Since then, two papers have been published on this topic. In one, Carlyon et al. (2009) reported that a narrower band noise was perceived as longer when it was preceded by a wider band noise of the same spectral density. In the other one, Sasaki et al. (2010)

\footnotetext{
T. Kuroda $(\bowtie)$

Japan Society for the Promotion of Science / Graduate School of Medical Sciences, Kyushu University, 3-1-1, Maidashi, Higashiku, Fukuoka 812-8582, Japan

e-mail: tkuroda@neurophy.med.kyushu-u.ac.jp

S. Grondin

Université Laval, Quebec City, Canada
}

systematically investigated the nature of the phenomenon with a sine tone preceded by a noise and named it the timestretching illusion.

Whereas no clear explanation has yet been established regarding the occurrence of the time-stretching illusion, Carlyon et al. (2009) and Sasaki et al. (2010) posited that the time-stretching illusion depends on the same mechanism as another auditory illusion, the continuity illusion (Elfner \& Caskey, 1965; Miller \& Licklider, 1950; Thurlow, 1957). The continuity illusion typically takes place in stimulus patterns where a sine tone and a noise are alternated repeatedly (Fig. 1b). When the noise is sufficiently more intense than the tone, the tone is perceived as continuing through the noise, and the stimulus pattern is perceived as consisting of a continuous tone overlapped with an intermittent noise.

While the continuity illusion disappears when the tone and the noise are far from each other in frequency, another effect is observed in this situation: The tone is perceived as longer when compared with the one presented without the noise (Warren, Bashford, Healy, \& Brubaker, 1994). The overestimation of the tone's duration gets larger as the tone and the noise get closer in frequency. When the overestimation reaches a maximum - that is, when both sounds are in the same frequency region - the tone is perceived as continuing through the noise, resulting in the continuity illusion. Because the overestimation is observed before the completion of the continuity illusion, it is referred to as an incomplete continuity illusion. The incomplete continuity illusion was demonstrated with sound patterns consisting of a noise and a tone repeated alternately; however, the overestimation could take place even when the noise and the tone were presented only once. In other words, the time-stretching illusion could be viewed as an example of the incomplete continuity illusion.

Whereas different approaches have been taken to explain the occurrence of the continuity illusion (Ciocca \& Bregman, 


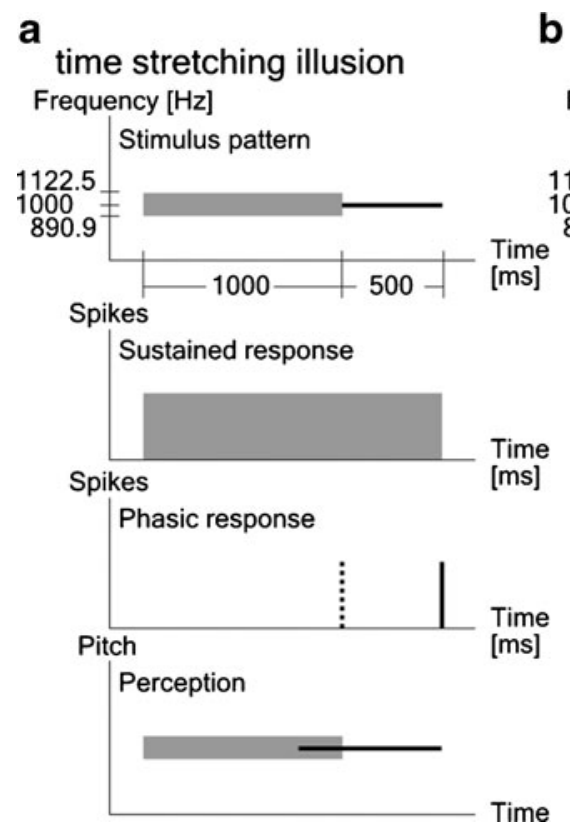

b
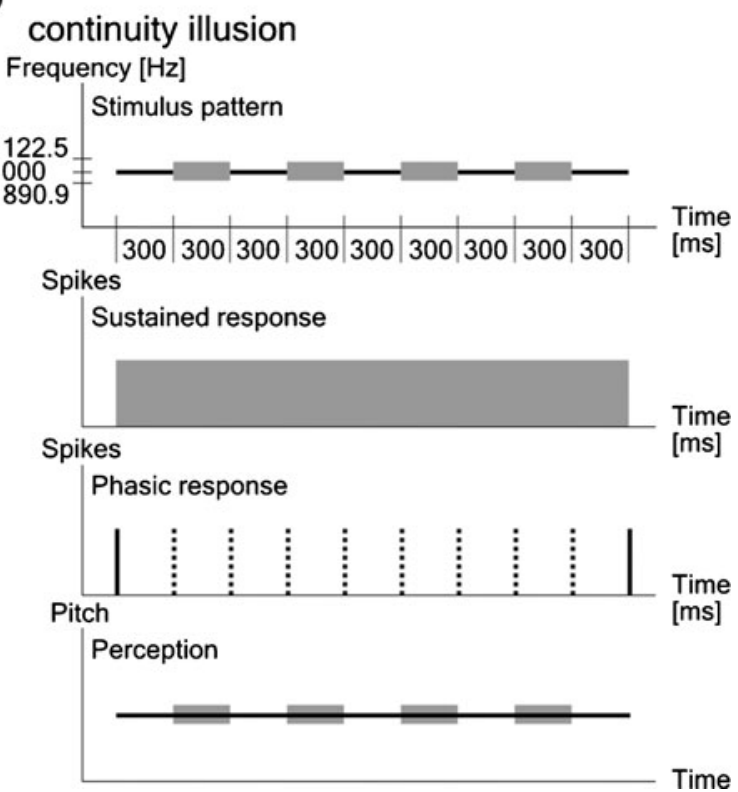

Fig. 1 The time-stretching illusion (a) and the continuity illusion (b), with the expected excitation pattern of sustained and phasic responses to sine tones' frequency. In the phasic excitation, solid lines indicate the responses expected to appear, while dashed lines indicate the responses expected to disappear

1987; Drake \& McAdams, 1999; Houtgast, 1972; Riecke, Esposito, Bonte, \& Formisano, 2009; Warren, Obusek, \& Ackroff, 1972), a recent neurophysiological study by Petkov, O'Connor, and Sutter (2007) indicated that the difficulty of detecting sound edges (i.e., onsets and terminations), which was originally proposed by psychological studies (Bregman, 1990; Bregman \& Dannenbring, 1977), is related to the occurrence of the continuity illusion (see also Haywood, Chang, \& Ciocca, 2011, for recent psychological approaches). They presented macaque monkeys a sound pattern that was expected to cause the continuity illusion and recorded the excitation of two types of neurons included in the primary auditory cortex: One, called sustained-response neurons, responds continuously during the presentation of sounds, while the other, called phasic-response neurons, responds transiently to temporal changes such as the onset and termination of sounds. They reported that, when a discontinuous tone was presented in isolation (without a noise), the phasic neurons responded to the termination and the onset that bounded the gap of the discontinuous sound. However, when a noise was inserted into the gap of the discontinuous tone (i.e., in the illusion pattern), the phasic neurons failed to respond to the termination and the onset that bounded the missing part of the discontinuous sound. They also found that in the illusion pattern, the sustained neurons that could respond to the discontinuous sound also continued excitation during the missing part of the discontinuous sound.

Indeed, Sasaki et al. (2010) attributed the occurrence of the time-stretching illusion to the failure to detect the onset of the following sound. They argued that, when a tone is preceded by a more intense noise, the detection of the tone's onset is disturbed, resulting in an illusory restoration of the missing onset within the preceding noise. They did not clearly explain why the missing onset is restored within the preceding noise instead of at its original position. However, it seemed plausible that listeners are biased to perceive the tone as having begun before the termination of the preceding noise because, in addition to the failure to detect the tone's onset, the preceding noise also stimulates the sustained-response neurons that can respond to the tone.

We conducted an experiment where the constant method was used to examine whether the time-stretching illusion would also take place when a sine tone is followed by a noise. The following noise was expected to disturb the detection of the tone's termination. Carlyon et al. (2009) and Sasaki et al. (2010) employed only one type of sound configuration where a tone was preceded by a noise, whereas we employed two more types of configuration. In one type, a tone was followed by a noise. In the other type, a tone was located between two consecutive noises. The noise was $6 \mathrm{~dB}$ more intense or $6 \mathrm{~dB}$ weaker than the tone. Sasaki et al. (2010) reported that the timestretching illusion was strengthened (i.e., overestimation increased) when the preceding noise was made more intense. Thus, we examined from two viewpoints the occurrence of the time-stretching illusion with a following noise: (1) whether a tone would be perceived as longer when it was followed by a more intense noise than when presented in isolation and (2) whether increasing the following-noise intensity (from -6 to + $6 \mathrm{~dB}$ ) relative to the tone intensity would result in a longer perceived duration of the tone. 


\section{Method}

Participants

Thirty-two participants were recruited. They were Université Laval students or employees with normal hearing, 19 males and 13 females, 18-40 years of age. They consented to their participation by signing a form approved by the institutional ethical committee and received $\$ 60 \mathrm{CAN}$ for their participation. $^{1}$

Apparatus and stimuli

Digital signals of stimuli were sampled at $44100 \mathrm{~Hz}$ and quantized to 16 bits. The stimuli were delivered from headphones (Sennheiser HD 477) connected to a computer (IBM Netvista). Each trial consisted of the standard tone and the comparison tone, which were successively presented. They were sinusoidal tones of $1000 \mathrm{~Hz}$ at about $60 \mathrm{dBA}$. The standard tone was fixed at $500 \mathrm{~ms}$, and the comparison tone was varied from 360 to $640 \mathrm{~ms}$ in steps of $40 \mathrm{~ms}$. The standard tone and the comparison tone were presented in two types of order: The standard was presented before the comparison tone (standard-comparison order), and vice versa (comparison-standard order).

The comparison tone was always presented in isolation. The standard tone was presented in one of four types of arrangement (Fig. 2). (1) In the noise-tone condition, which is the typical time-stretching pattern, the tone was preceded by a noise. (2) In the tone-noise condition, the tone was followed by a noise. (3) In the noise-tone-noise condition, the tone was located between two consecutive noises. (4) In the control condition, the tone was presented in isolation. The noise was a 500-ms 1/3-octave band-pass noise with a center frequency of $1000 \mathrm{~Hz}$ and was $6 \mathrm{~dB}$ more intense (the $+6-\mathrm{dB}$ condition) or $6 \mathrm{~dB}$ weaker (the $-6-\mathrm{dB}$ condition) than the tone.

The standard pattern and the comparison tone were separated with an interstimulus interval that was randomly varied from 2,000 to 2,500 ms. To avoid spectral splatter, the amplitude rose and decayed during $10 \mathrm{~ms}$ at the beginning and at the end of each sound with raised-cosine ramps. The rise and decay times were included in the sounds' length.

\section{Procedure}

The task was performed by clicking on panes on a computer display. Participants listened to the stimulus pair (standard and comparison) by clicking on the "play" pane. A silent interval of 2.0-2.5 s began right after clicking, and then the first

\footnotetext{
1 Three more participants were recruited, but the data from these participants were not kept for the analyses. These participants showed poor goodness of fitting the cumulative normal distribution to some psychometric functions $\left(R^{2}\right.$ values of less than .50$)$.
}

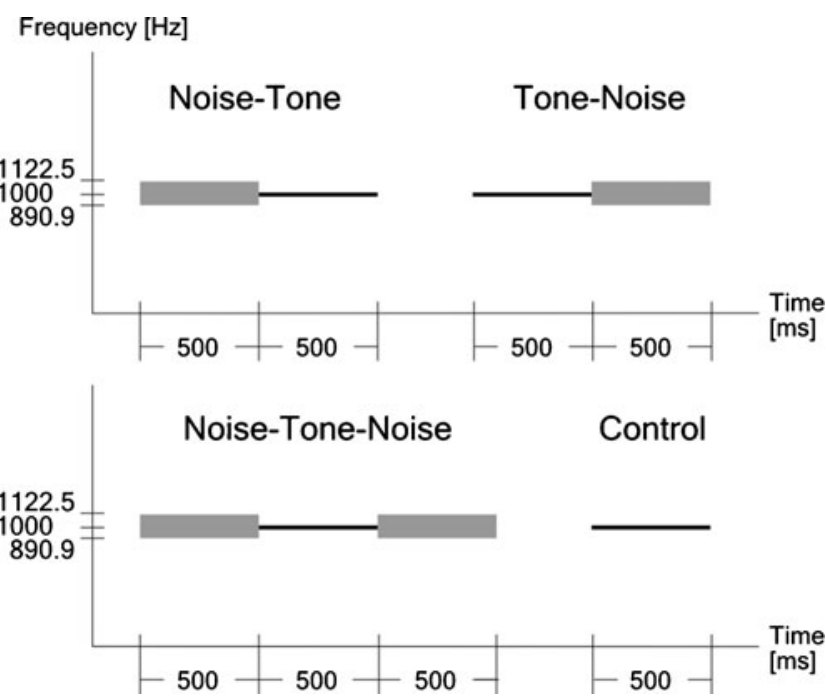

Fig. 2 Standard patterns

stimulus pattern was presented. The participants were allowed to listen to the stimulus pair only once on each trial, but when listening was disturbed for some specific reason (e.g., yawning or coughing), they could listen again to the pair by clicking on the "replay" pane.

Participants were told that each trial consisted of two successive tones of clear timbre (i.e., sine tones), which were sometimes accompanied with a sound or sounds of noisy timbre (i.e., noises). Participants judged whether the second tone was "shorter" or "longer" than the first tone by clicking on panes. ${ }^{2}$ They could answer that the two tones were "equal" in duration. They could also answer that they were "unsure" whether the second tone was shorter or longer than the first one but perceived these tones as different durations. Participants were instructed not to use the last two alternatives except when definitely necessary.

While the configuration conditions were compared with a within-participants design, the intensity conditions were compared with a between-participants design. Indeed, 16 out of the 32 participants were assigned to the $+6-\mathrm{dB}$ condition, and the other 16 were assigned to the $-6-\mathrm{dB}$ condition. The experiment consisted of six sessions for both participant groups. In each group, 8 participants completed three sessions in the standard-comparison order and then three sessions in the comparison-standard order. The other (8) participants completed these sets of sessions in the opposite order. Each session consisted of four blocks, one for each configuration condition (noise-tone, tone-noise, noise-tone-noise, and control). The order of these blocks was counterbalanced. Each block consisted of 80 trials where eight comparison

\footnotetext{
$\overline{2}$ The computer program did not receive participants' response before the end of presentation of stimuli, in order to avoid the possibility that participants would make a judgment before the end of a noise that followed a tone when the standard pattern was presented after the comparison pattern.
} 
intervals were presented 10 times each in random order. Two practice trials, where randomly selected comparison intervals were presented, were carried out at the beginning of each block. Each session took about an hour, and each participant completed one session per day.

\section{Results}

The alternative responses of "equal" and "unsure" were split between the "shorter" and the "longer" responses. Eight-point psychometric functions were constructed for each participant by calculating the probability of perceiving the second (comparison) tone as "longer" in the standard-comparison order and the probability of perceiving the second (standard) tone as "shorter" in the comparison-standard order as a function of the comparison interval. These types of probabilities meant how frequently participants perceived the comparison interval as longer than the standard one. Each point of the functions was based on 30 responses ( 3 sessions $\times 10$ repetitions). The cumulative normal distribution was fit to the resulting functions. The goodness of fit was generally high: The $R^{2}$ value was above .90 in 225 cases out of 256 ( 2 intensities $\times 2$ orders $\times 4$ patterns $\times 16$ participants $)$ and was between .90 and .55 in 31 cases. An $x$-axis (comparison interval) value at which the curve crossed the probability of .50 was estimated from each function and was referred to as the point of subjective equality (PSE). A higher PSE indicated a longer perceived duration of the standard tone. The results of the standard-comparison order and of the comparison-standard order were averaged so that any order effects would be canceled out.

The box plots of PSEs are shown in Fig. 3. Because the results of each condition did not seem to conform to the normal distribution, we avoided using parametric tests when comparing the experimental conditions. Instead, we conducted Wilcoxon signed rank tests to examine whether the median in each condition is significantly different from $500 \mathrm{~ms}$ - that is, positing the null hypothesis that the median is $500 \mathrm{~ms}(N=16$ for each intensity group). The median was significantly higher than $500 \mathrm{~ms}$ for the noise-tone condition in the $+6-\mathrm{dB}$ condition $(p=.001)$ and even in the $-6-\mathrm{dB}$ condition $(p=.049)$. None of the other conditions led to significant differences $(p>.100)$. We then conducted Mann-Whitney tests to examine whether the median significantly differed between the $+6-$ and $-6-\mathrm{dB}$ conditions. The noise-tone condition led to significantly higher PSEs in the + $6-\mathrm{dB}$ than in the $-6-\mathrm{dB}$ condition $(p=.005)$. None of the other conditions led to significant differences $(p>.100)$.

The scatterplots of PSEs are shown in Fig. 4. The correlations between the conditions were examined with Spearman's rank correlation coefficients ${ }^{3}$; the results are also apparent in

\footnotetext{
3 The reason for using nonparametric correlation coefficients is that the PSEs of 2 participants were much higher (above $630 \mathrm{~ms}$ ) than those of the other participants in the noise-tone-noise condition at $+6 \mathrm{~dB}$.
}

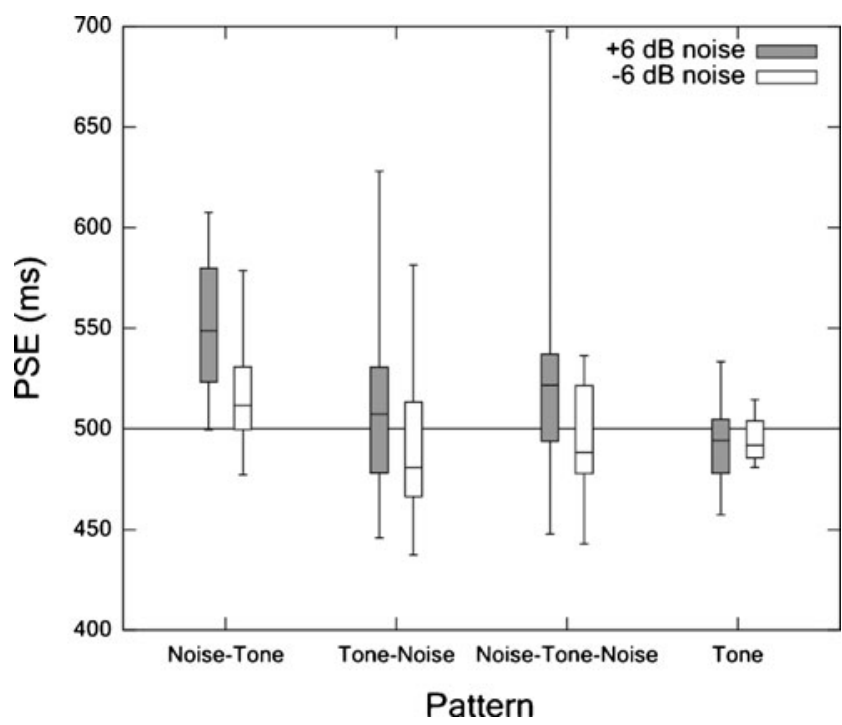

Fig. 3 Box plots of the point of subjective equality (PSE) in each experimental condition. Each box consists of a minimum, a lower quartile, a median, an upper quartile, and a maximum value

this figure. Neither of the two intensity conditions led to significant correlations between the noise-tone and the tonenoise conditions. Significant correlations were found only between the tone-noise and the noise-tone-noise conditions at $+6 \mathrm{~dB}$ and between the noise-tone and the noise-tonenoise conditions at $-6 \mathrm{~dB}$.

\section{Discussion}

No signs of the time-stretching illusion could be observed when a tone was followed by a noise. The duration of a tone was overestimated when it was preceded by a noise, but not when followed by a noise or when located between two consecutive noises. The increasing of the noise intensity resulted in larger overestimation, but only when a tone was preceded by a noise. Given that there were no correlations between the results for the noise-tone and the tone-noise conditions, it seemed difficult to explain the present results only with the potential failure to detect the tone's edges, as explained in the introduction.

The absence of time stretching with a following noise might be related to functional differences between onsets and terminations in the auditory system. While several studies of auditory organization regarded sound terminations, as well as onsets, as one type of the elements that could be organized perceptually (Crum \& Bregman, 2006; Kuroda, Nakajima, \& Eguchi, 2012; Nakajima et al., 2000), it is known that acoustic terminations, as compared with onsets, have a weak effect on inducing neurophysiological responses and determining the perceptual timbre of sounds. Phillips, Hall, and Boehnke (2002) indicated that the auditory nerve system (brainstem) is elaborated to respond to onset cues, but not to termination cues. Campbell (1994) and 


\section{$+6 \mathrm{~dB}$ noise}

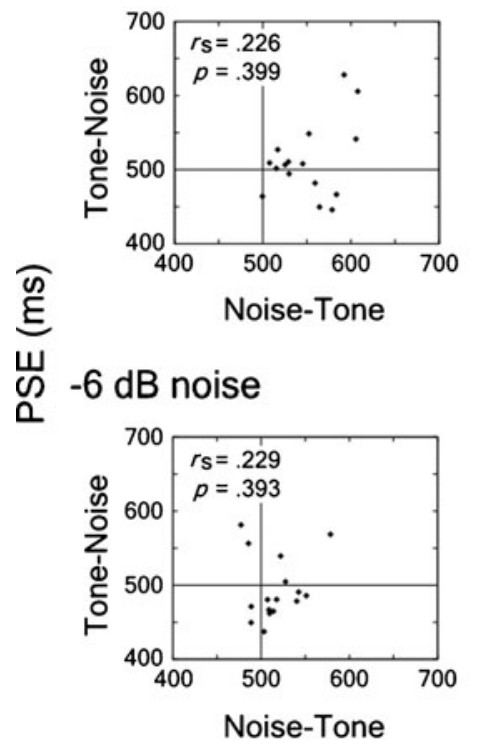

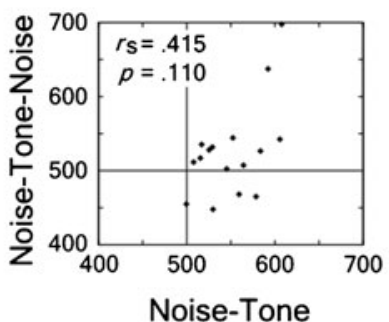
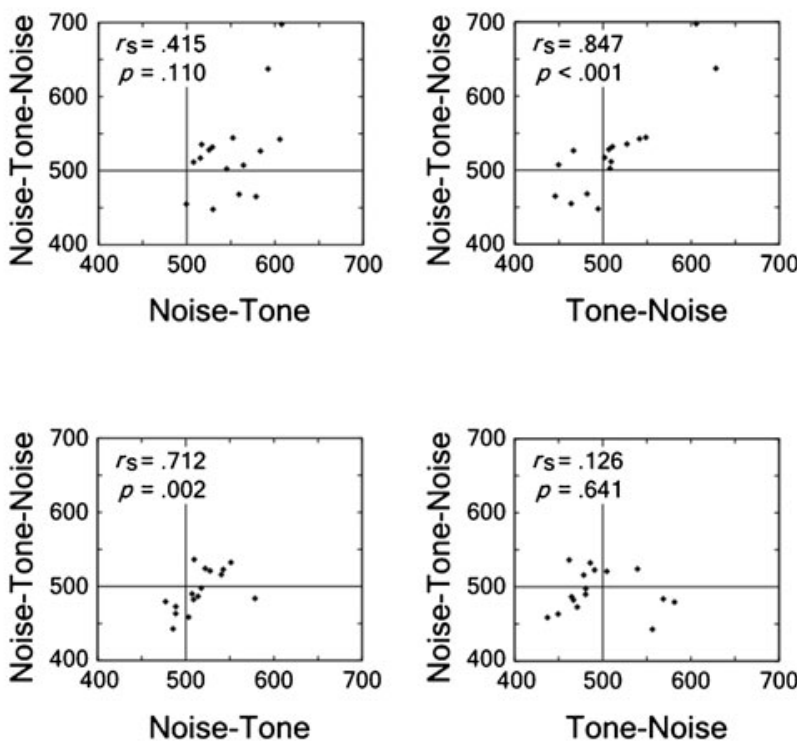

PSE (ms)

Fig. 4 Scatterplots of point of subjective equality (PSE) for each pair of experimental conditions (excluding the control) for the +6-dB noise (upper panel) and for the $-6-\mathrm{dB}$ noise (lower panel)

Gordon (1987) indicated that the steepness of onsets (the rise time) determines sound timbre, while no studies have yet shown that the steepness of terminations (the decay time) determines timbre (see also Bregman, Ahad, \& Kim, 1994, for the onset vs. termination differences in pitch identification). In the present study, no effect was observed when adding a following noise to disturb the detection of the tone's termination. However, it should be noticed that a tone between two consecutive noises did not result in any overestimation. This tone should have been perceived as being of at least the same duration as a tone that was only preceded by a noise, if the preceding noise had disturbed the detection of the tone's onset. Since the preceding noise did not contribute to the increase of the tone's duration when the following noise was also presented, the following noise might have canceled out the preceding noise's effect.

One might argue that adding a noise near a tone resulted in the change of the tone's loudness, which could affect its perceived duration. Indeed, several studies have indicated that the loudness of a sound can be decreased when it is presented after a more intense sound (induced loudness reduction or loudness recalibration; see Epstein, 2007; Marozeau \& Epstein, 2008). However, it seemed difficult to explain the time-stretching illusion by such loudness change, because the illusion could take place even when the noise had the same intensity as the tone and, moreover, could remain when the noise was weaker than the tone (Sasaki et al., 2010). Indeed, there was a slight but significant overestimation even when a tone was preceded by a 6-dB weaker noise in the present study.

Whereas how loudness (or intensity) affects perceived duration has not yet been established in the literature (see Berglund,
Berglund, Ekman, \& Frankehaeuser, 1969; Eisler \& Eisler, 1992), a recent study by Matthews, Stewart, and Wearden (2011) used the stimuli similar to the ones used in the present study and indicated that the relative intensity of targets and backgrounds, instead of the absolute intensity of targets, is a crucial factor for determining perceived duration. In their study, a standard sound of $600 \mathrm{~ms}$ and a comparison sound of variable duration were successively presented with a background sound of about $42 \mathrm{dBA}$ (quiet) or $72 \mathrm{dBA}$ (loud), which was presented before the standard, between the standard and the comparison, and after the comparison. The standard and the comparison were 46 and $68 \mathrm{dBA}$ or 68 and $46 \mathrm{dBA}$, respectively. When the standard/comparison was $46 / 68 \mathrm{~dB}$, as compared with $68 /$ $46 \mathrm{~dB}$, the probability of perceiving the comparison as longer than the standard was increased in the quiet background but was decreased in the loud background. In other words, more intense comparisons were perceived as longer when the standard and comparison intensity was above the (quiet) background intensity, while weaker comparisons were perceived as longer when the standard and comparison intensity was below the (loud) background intensity. Whereas the authors did not discuss much about whether the quiet or the loud background led to a longer perceived duration of the comparison (of the same intensity), we could read from their data that, as compared with the quiet background, the loud background resulted in an overestimation of the 46- $\mathrm{dB}$ comparison but an underestimation of the $68-\mathrm{dB}$ comparison duration. The result of the 68-dB comparison did not seem consistent with what occurs in the time-stretching illusion, where the increasing of the background intensity results in a longer perceived duration 
of the tone, if we regard the preceding noise as the background. It is therefore difficult to posit that exactly the same mechanism underlies the time-stretching illusion and the relative intensity effects between the target and the background.

In summary, the time-stretching illusion takes place only when a tone is preceded by a noise. This time-stretching illusion has been compared with the continuity illusion, which is an important issue in the field of auditory organization. In addition to providing information for auditory organization, the time-stretching illusion probably also reveals critical information about the mechanisms involved in the processing of temporal information. Further investigations about what causes the time-stretching illusion will likely lead to theoretical advances in both fields, time perception and auditory organization.

Author Notes This research was made possible by a research grant awarded to S.G. by the Natural Sciences and Engineering Council of Canada. We would like to extend special thanks to Catherine LussierLévesque, Elsa Massicotte, Félix Désautels, Flore Morneau-Sévigny, Katherine Labonté, Larissa Roy, Noémie de la Sablonnière, and Vincent Laflamme for their help in data collection and to Stephen Handel, Yoshitaka Nakajima, and an anonymous reviewer for valuable suggestions on an earlier version of the manuscript. All correspondence should be addressed to: Tsuyoshi Kuroda, Graduate School of Medical Sciences, Kyushu University, 3-1-1, Maidashi, Higashiku, Fukuoka, 812-8582, Japan. E-mail: tkuroda@neurophy.med.kyushu-u.ac.jp.

\section{References}

Berglund, B., Berglund, U., Ekman, G., \& Frankehaeuser, M. (1969). The influence of auditory stimulus intensity on apparent duration. Scandinavian Journal of Psychology, 10, 21-26.

Bregman, A. S. (1990). Auditory scene analysis: The perceptual organization of sound. Cambridge, MA: MIT Press.

Bregman, A. S., Ahad, P. A., \& Kim, J. (1994). Resetting the pitchanalysis system. 2. Role of sudden onsets and offsets in the perception of individual components in a cluster of overlapping tones. Journal of the Acoustical Society of America, 96, 2694-2703.

Bregman, A. S., \& Dannenbring, G. L. (1977). Auditory continuity and amplitude edges. Canadian Journal of Psychology, 31, 151-159.

Campbell, S. L. (1994). Uni- and multidimensional identification of rise time, spectral slope, and cutoff frequency. Journal of the Acoustical Society of America, 96, 1380-1387.

Carlyon, R. P., Deeks, J. M., Shtyrov, Y., Grahn, J., Gockel, H. E., Hauk, O., \& Pulvermüller, F. (2009). Changes in the perceived duration of a narrowband sound induced by a preceding stimulus. Journal of Experimental Psychology: Human Perception and Performance, $35,1898-1912$.

Ciocca, V., \& Bregman, A. S. (1987). Perceived continuity of gliding and steady-state tones through interrupting noise. Perception \& Psychophysics, 42, 476-484.

Crum, P. A. C., \& Bregman, A. S. (2006). Effects of unit formation on the perception of a changing sound. Quarterly Journal of Experimental Psychology, 59, 543-556.
Drake, C., \& McAdams, S. (1999). The auditory continuity phenomenon: Role of temporal sequence structure. Journal of the Acoustical Society of America, 106, 3529-3538.

Eisler, H., \& Eisler, A. D. (1992). Time perception: Effects of sex and sound intensity on scales of subjective duration. Scandinavian Journal of Psychology, 33, 339-358.

Elfner, L., \& Caskey, W. E. (1965). Continuity effects with alternately sounded noise and tone signals as a function of manner of presentation. Journal of the Acoustical Society of America, 38, 543-547.

Epstein, M. (2007). An introduction to induced loudness reduction. JASA Express Letters, 122, EL74-EL80.

Gordon, J. W. (1987). The perceptual attack time of musical tones. Journal of the Acoustical Society of America, 82, 88-105.

Haywood, N. R., Chang, I.-C., \& Ciocca, V. (2011). Perceived tonal continuity through two noise bursts separated by silence. Journal of the Acoustical Society of America, 130, 1503-1514.

Houtgast, T. (1972). Psychophysical evidence for lateral inhibition in hearing. Journal of the Acoustical Society of America, 51, 18851894.

Kuroda, T., Nakajima, Y., \& Eguchi, S. (2012). Illusory continuity without sufficient sound energy to fill a temporal gap: Examples of crossing glide tones. Journal of Experimental Psychology: Human Perception and Performance, 38, 1254-1267.

Marozeau, J., \& Epstein, M. (2008). Induced loudness reduction as a function of frequency difference between test tone and inducer. Perception \& Psychophysics, 70, 725-731.

Matthews, W. J., Stewart, N., \& Wearden, J. H. (2011). Stimulus intensity and the perception of duration. Journal of Experimental Psychology: Human Perception and Performance, 37, 303-313.

Miller, G. A., \& Licklider, J. C. R. (1950). The intelligibility of interrupted speech. Journal of the Acoustical Society of America, 22, 167-173.

Nakajima, Y., Sasaki, T., Kanafuka, K., Miyamoto, A., Remijn, G., \& ten Hoopen, G. (2000). Illusory recouplings of onsets and terminations of glide tone components. Perception \& Psychophysics, 62, 14131425 .

Petkov, C. I., O’Connor, K. N., \& Sutter, M. L. (2007). Encoding of illusory continuity in primary auditory cortex. Neuron, 54, 153-165.

Phillips, D. P., Hall, S. E., \& Boehnke, S. E. (2002). Central auditory onset responses, and temporal asymmetries in auditory perception. Hearing Research, 167, 192-205.

Riecke, L., Esposito, F., Bonte, M., \& Formisano, E. (2009). The timing of sensory-perceptual transformations in auditory cortex. Neuron, $64,550-561$.

Sasaki, T., Nakajima, Y., \& ten Hoopen, G. (1993). The effect of a preceding neighboring tone on the perception of filled durations. Proceedings of the Acoustical Society of Japan, spring meeting [in Japanese] (pp. 347-348). Tokyo: Acoustical Society of Japan.

Sasaki, T., Nakajima, Y., ten Hoopen, G., van Buuringen, E., Massier, B., Kojo, T., ... Ueda, K. (2010). Time stretching: Illusory lengthening of filled auditory durations. Attention, Perception, \& Psychophysics, 72, 1404-1421.

ten Hoopen, G., Miyauchi, R., \& Nakajima, Y. (2008). Time-based illusions in the auditory mode. In S. Grondin (Ed.), Psychology of time (pp. 139-187). Bingley, U.K.: Emerald Group Publishing.

Thurlow, W. R. (1957). An auditory figure-ground effect. American Journal of Psychology, 70, 653-654.

Warren, R. M., Bashford, J. A., Jr., Healy, E. W., \& Brubaker, B. S. (1994). Auditory induction: Reciprocal changes in alternating sounds. Perception \& Psychophysics, 55, 313-322.

Warren, R. M., Obusek, C. J., \& Ackroff, J. M. (1972). Auditory induction: Perceptual synthesis of absent sounds. Science, 176, 11491151. 